\title{
Within the Enemy's Camp: contribution of the granuloma to the dissemination, persistence and transmission of Mycobacterium tuberculosis
}

\author{
Christopher R. Shaler, Carly N. Horvath, Mangalakumari Jeyanathan and Zhou Xing* \\ McMaster Immunology Research Centre, Department of Pathology and Molecular Medicine, McMaster University, Hamilton, ON, Canada
}

Edited by:

Stephen Wesley Chensue, University of Michigan, USA

\section{Reviewed by:}

Junji Yodoi, Kyoto University, Japan Kottarappat Dileepan, The University of Kansas Medical Center, USA

\section{*Correspondence:}

Zhou Xing, McMaster Immunology Research Centre, Department of Pathology and Molecular Medicine, McMaster University, MDCL-Room 4012, 1280 Main Street West, Hamilton, ON, Canada L8S 4K1. e-mail:xingz@mcmaster.ca

\begin{abstract}
Pulmonary tuberculosis, caused by Mycobacterium tuberculosis (M.tb) represents a leading global health concern, with 8.7 million newly emerging cases, and 1.4 million reported deaths annually. Despite an estimated one third of the world's population being infected, relatively few infected individuals ever develop active clinical disease. The ability of the host to remain latently infected while preventing disease is thought to be due to the generation of a robust type 1 immune response in the lung, capable of controlling, but not clearing, M.tb. A key feature of the type 1 immune response to $M . t b$ is the formation of immune cellular aggregates termed granuloma. The granuloma structure has long been considered a hallmark of host's protective response toward M.tb. Historically, a correlative relationship between granuloma formation/maintenance and bacterial control has been seen in models where disrupted granuloma formation or structure was found to be fatal. Despite this established relationship much about the granuloma's role in M.tb immunity remains unknown. Recent publications suggest that the granuloma actually aids the persistence of M.tb and that the development of a necrotic granuloma is essential to person-to-person transmission. Our group and others have recently demonstrated that enclosed within the granuloma is a population of immunologically altered antigen-presenting cells and T lymphocyte populations. Of note, the ability of these populations to produce type 1 cytokines such as interferon-gamma, and bactericidal products including nitric oxide, are significantly reduced, while remaining competent to produce high levels immunosuppressive interleukin-10. These observations indicate that although the chronic granuloma represents a highly unique environment, it is more similar to that of a tumor than an active site of bacterial control. In this review we will explore what is known about this unique environment and its contribution to the persistence of M.tb.
\end{abstract}

Keywords: tuberculosis, immunopathology, immune regulation, granuloma, bacterial persistence, mycobacteria

\section{INTRODUCTION}

Pulmonary tuberculosis (TB) caused by infection with Mycobacterium tuberculosis (M.tb) is the leading cause of death due to a bacterial pathogen and is responsible for 1.4 million deaths annually, latently infecting one third of the world's population (WHO, 2012). Despite the magnitude of individuals infected, the rate of mortality is relatively low with approximately $90 \%$ infected individuals controlling, but not clearing, M.tb (WHO, 2012). The ability of the host to "control" M.tb infection encompasses a number of immunological processes designed to restrain bacterial dissemination and persistence, and reduce person-to-person transmission. The classical hallmark of anti-TB host defense is the formation of type 1 immune granuloma in the lung. Historically, the granuloma has been perceived as essential to anti-TB host defense as the host is incapable of sterile clearance and thus is forced to segregate the infected cells as a means to preserve itself. However, experimentally little is known of the role of the granuloma in bacterial control.

\section{THE HISTORICAL VIEW OF MYCOBACTERIAL GRANULOMA}

First described in 1679, pathologists discovered unique structures in the lungs of TB patients (de le Boe, 1679; reviewed in Ramakrishnan, 2012). These structures were then termed tubercles and represent what we now know as granulomas. Commonly, it was observed that persons who had died of TB had a large number of these distinct pathological lesions, and the presence of tubercles became an associated hallmark of active TB disease. It was not until 1884 that tubercles were also characterized in individuals who had died from diseases other than TB. Upon post-mortem examination, a number of these individuals had lung lesions (granulomas) containing live TB bacilli, giving the first indication that TB latency may relate to the formation of granuloma (Dejerine, 1884). However, upon further microbiological examination, it was revealed that live bacilli persisted not only within the granuloma itself, but also in the surrounding lung tissue, albeit to a lesser degree (Wang, 1916; Opie and Aronson, 1927; Robertson, 1933; Feldman and Baggenstoss, 1938, 1939). It was around this time that the 
protective view of the granuloma began to gain public acceptance and it was proposed that the recruitment of activated lymphocytes and the formation of a lymphocytic cuff served to wall-off infected macrophages as a means of limiting dissemination. However, the role of granuloma in TB has remained enigmatic largely because of the unavailability of reliable animal models and appropriate techniques to observe the dynamic process of granuloma evolution. Although different experimental models (mice, guinea pig, rabbit, cattle, and macaque) have been developed, only cattle and macaque monkeys form the type of granuloma that closely resembles those seen in humans (Capuano et al., 2003; Flynn et al., 2003; Tsai et al., 2006; Hunter et al., 2007; Via et al., 2008). Despite being extensively used as a model of $\mathrm{TB}$, the murine granuloma lacks many of the unique characteristic features of the human granuloma including centralized necrosis, giant multinuclear cells, and a defined "lymphocytic cuff" (Rhoades et al., 1997). Contrasting the classical notion of its protective role, a number of recent studies have demonstrated unaltered bacterial control even in the absence of granuloma formation, strongly arguing against the granuloma being essential to bacterial restriction (Johnson et al., 1998; Scott and Flynn, 2002; Pearl et al., 2004). Moreover, it is now known that the Mycobacterium can significantly alter the immune environment of the granuloma as means to facilitate its persistence (Ly et al., 2007; Scott-Browne et al., 2007; Marino et al., 2010; Castano et al., 2011; O'leary et al., 2011). Regardless, the common perception remains that the granuloma serves to limit bacterial growth and prevent dissemination by segregating infected cells, and the role of the granuloma in M.tb infection remains an issue of continued debate. In this review we will challenge the traditional view of the function of granuloma, exploring what is known about its progression and maturation, and how this unique environment may in fact contribute to the persistence and transmission of M.tb.

\section{FORMATION OF THE TYPE 1 IMMUNE GRANULOMA DURING M.tb INFECTION}

The formation of granuloma is a dynamic process that begins shortly after infection and continuously evolves over time. Temporally, the granuloma can be divided into three distinct phases: (1) the "innate granuloma," a loose aggregate composed primarily of recruited macrophages and neutrophils; (2) the "immune granuloma" formed following the emergence of antigen-specific T cells; and (3) the "chronic granuloma," resulting from distinct morphological changes in granuloma structure (Figure 1).

\section{FORMATION OF THE "INNATE GRANULOMA"}

Shortly after aerosol exposure, M.tb infects the resident alveolar macrophage (AM) initiating the early inflammatory response. While amplifying the host immune response, the recruitment of innate immune cells inadvertently provides a large number of new targets for M.tb to infect and is thought to contribute to the early dissemination of M.tb (Doenhoff, 1997; Davis and Ramakrishnan, 2009). Augmenting this problem, the infected AM is unable to kill internalized mycobacteria due to impaired phagolysosome fusion, a process essential to the destruction of the phagocytosed bacteria (Welin et al., 2011). The efficiency by which a Mycobacterium species arrests phagolysosome fusion is directly attributable to its relative virulence, with highly virulent strains such as M.tb almost completely inhibiting fusion (Ferrer et al., 2010). Incapable of killing internalized M.tb, infected macrophages secrete an array of pro-inflammatory and chemoattractant cytokines including tumor necrosis factor (TNF), interleukin (IL)-6, and IL-8 which facilitate the recruitment of new macrophages and granulocytes to the site of infection and lead to the formation of the "innate granuloma" (Birkness et al., 2007). This initial recruitment is essential to establishing the macrophage-dominated center of the "immune" granuloma.

\section{CONTRIBUTION OF M.tb TO THE FORMATION OF THE "INNATE GRANULOMA"}

Historically the formation of granuloma has been considered to be a host-mediated event. Using an M. marinum model, real-time microscopic visualization has challenged this notion, revealing that virulent Mycobacterium drives the nascent formation of the early granuloma. A number of elegant studies conducted by Ramakrishnan's group have demonstrated the unique interplay between the Mycobacterium and the host immune system in the early stage of granuloma formation. To this end, the early release of $6 \mathrm{kDa}$ early secretory antigenic target (ESAT-6) by M. marinum led to the activation of the epithelium, which facilitated the recruitment of macrophages to the site of infection through inducing the production of matrix metalloproteinase-9 (MMP-9) (Davis and Ramakrishnan, 2009; Volkman et al., 2010). In murine models, M.t $b$ was also found to drive MMP-9 expression. Thus either broad MMP inhibition or MMP-9-specific depletion delayed granuloma formation, resulting in impaired macrophage recruitment to the site of infection and reduced granuloma size (Taylor et al., 2006). These findings indicate that M.tb may actually promote granuloma formation and utilizes the structure for its own benefit. Furthermore, it was demonstrated that virulent mycobacteria can utilize the innate granuloma as means of recruiting target cells allowing for the early dissemination of mycobacteria throughout the host (Davis and Ramakrishnan, 2009). This view is supported further by the observation that the early granuloma is not a static environment and there is a significant movement of antigen-presenting cell (APC) populations into and out of the early granuloma (Chiu et al., 2004; Egen et al., 2008, 2011; Schreiber et al., 2011).

\section{FORMATION OF THE "IMMUNE GRANULOMA"}

Following innate activation, dendritic cells are recruited to the lung and transport mycobacteria or mycobacterial antigens to the mediastinal lymph node (MLN). Within the MLN antigenloaded APCs activate antigen-specific T cells. Due to the nature of M.tb infection, the majority of bacilli and antigen reside within an endosome, and are most efficiently loaded onto major histocompatibility complex (MHC) class II (Mogues et al., 2001; Anis et al., 2008; Yahagi et al., 2010). The loading of MHC class II facilitates the priming of Th1 interferon-gamma (IFN- $\gamma$ )-secreting $\mathrm{T}$ cells, which rapidly home to the lung. While the dominant subset of $\mathrm{T}$ cells are $\mathrm{CD} 4^{+}$, cross-presentation also allows for the strong induction of $\mathrm{CD}^{+} \mathrm{T}$ cells, collectively generating a type 1 polarized adaptive immune response (Winau et al., 2006). Although outside the scope of this review, it should be noted that there is a substantial lag period between M.tb infection and the initiation of antigen-specific T cell responses (reviewed in Shaler et al., 2012). 


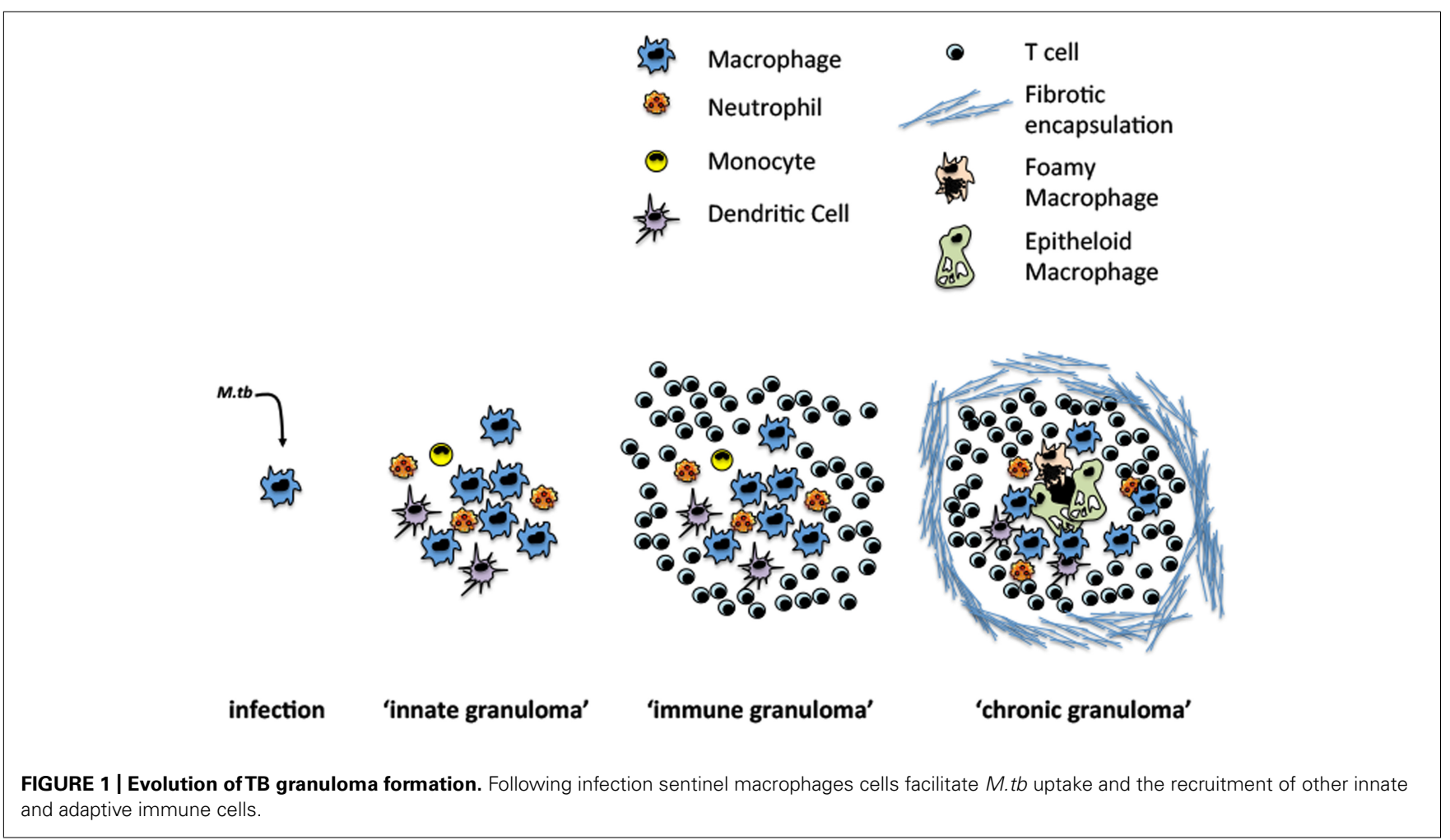

The continuous production of chemokines by infected lung APCs efficiently recruits newly primed $\mathrm{T}$ cells into the lung. Once in the lung, recruited $\mathrm{T}$ cells surround and wall-off infected macrophages, activate them for enhanced bactericidal function, and physically limit their mobility to restrain bacterial dissemination. Indeed, the arrival of effector $\mathrm{T}$ cells and the establishment of the classical "immune granuloma" is associated with a plateau in bacterial growth (Mogues etal., 2001). While the prevailing immune response generated following $M$.tb infection is highly similar between the mouse and man, the structural formation of the "immune granuloma" differs significantly. In mice, many of the hallmark features of the human granuloma are missing, and thus the knowledge from murine granuloma research should be interpreted with caution. Despite these limitations, animal models have provided significant insight, and have been invaluable in delineating the stages of granuloma formation.

\section{THE CONTROVERSIAL REQUIREMENT OF THE "IMMUNE GRANULOMA" IN LIMITING BACTERIAL DISSEMINATION}

In spite of the traditional protective view of granuloma, it has recently been revealed that the immune environment within granuloma is more conducive to M.tb persistence than its elimination. Despite the fact that animal models do not accurately replicate human granuloma structures, several murine studies have provided invaluable insight into the role of granuloma in preventing M.tb dissemination control. Early studies demonstrating the role of critical cytokines such as TNF and IFN- $\gamma$ perpetuated the notion that the granuloma was essential for bacterial segregation and limiting bacterial growth in the lung as the absence of either cytokine led to ill-formed granuloma and increased bacterial infection (Flynn et al., 1998; Kaneko et al., 1999; Algood et al., 2005; Beham et al., 2011; Gallegos etal., 2011). Moreover, any loss of $\mathrm{CD}^{+}{ }^{+} \mathrm{T}$ cell functionality results in a loss of the granuloma structure and extensive bacterial dissemination is seen in both man and mouse. While essential to the control of M.tb, the role of the $\mathrm{CD}^{+} \mathrm{T}$ cell in the granuloma's structure is somewhat species-dependent. Specifically, in humans, T cells surround and wall-off infected macrophages, and do not infiltrate the granuloma, but rather form a defined lymphocytic cuff. Conversely, murine $\mathrm{CD}^{+}{ }^{+} \mathrm{T}$ cells associate directly with infected cells infiltrating throughout the granuloma forming lymphocytic aggregates or pseudo-granulomas (Mogues et al., 2001; Tsai et al., 2006; Hunter et al., 2007; Via et al., 2008; Gallegos et al., 2011; Geldmacher et al., 2012). Nevertheless, while the loss of CD4 T cell-mediated immunity is detrimental to the host it is impossible to separate the relative contribution of the two processes to the impaired bacterial control: loss of Th1-mediated immunity and loss of granuloma structure, these studies suggest a critical role for the granuloma in preventing bacterial dissemination (Saunders et al., 2002; SegoviaJuarez et al., 2004). The essential role of CD4 T cells to the control of mycobacterial growth has largely been attributed to their potent IFN- $\gamma$ production and subsequent macrophage activation. While macrophage activation is essential to the control of M.tb in mouse and man, the role of nitric oxide has been an issue of some debate. Historically, studies have shown that human macrophages do not produce nitric oxide to the same degree in vitro as those isolated from mice (Aston et al., 1998). Recently, however, several groups have demonstrated that human macrophages from TB infected patients, as well as human macrophage cells lines are capable of inducing iNOS and producing nitric oxide in response 
to M.tb antigen (Rich et al., 1997; Jagannath et al., 1998; Dlugovitzky etal., 2000). Indeed, it appears that while the timing of nitric oxide and its role in TB control may differ somewhat between mouse and man, nitric oxide remains important in both species.

Recent studies suggest that the granuloma may be dispensable for preventing bacterial dissemination and may actually contribute to M.tb persistence. Moreover, in the absence of intracellular adhesion molecule-1 (ICAM-1), there is also a failure of granuloma formation, and despite this defect, mice are protected for the first 90 days post-infection, with no increase in bacterial growth compared to wildtype mice within this time frame (Johnson et al., 1998). Similarly, zebrafish models have shown that in the absence of early granuloma formation, there is no defect in the ability of the host to limit bacterial replication and dissemination, and that the granuloma may actually facilitate early dissemination (Volkman et al., 2004, 2010). Furthermore, in the absence of IL-27 in mice, there is a substantial defect in the ability of the host to form granuloma in response to M.tb infection, and yet infected mice exhibit markedly enhanced bacterial control when compared to their wildtype counterparts (Pearl et al., 2004). Indeed, recent studies indicate that granuloma does not always function to limit bacterial dissemination. For example, C-C chemokine receptor type 2 (CCR2) deficient mice form exaggerated granuloma structures when infected with M.tb and paradoxically have a decreased capacity to control bacterial growth (Scott and Flynn, 2002).

While it remains debatable whether the granuloma is required for bacterial control, growing evidence supports the notion that the fate of M.tb within the granuloma is situation-dependent. For example, the initial inoculum size may influence the number of macrophages and granulocytes that are recruited to the site of infection. If a large number of cells are initially recruited, spatially it becomes difficult for effector $\mathrm{T}$ cells to interact with the infected cells residing at the core of the granuloma limiting their ability to activate these centralized macrophages to kill internalized M.tb (Segovia-Juarez et al., 2004). In comparison, a small initial inoculum size infects a small number of cells at the core of granuloma, which may increase the likelihood of interaction of infected cells with effector T cells (Segovia-Juarez et al., 2004). Based on this notion, it is tempting to speculate that a stronger initial innate immune response may perpetuate the infection and limit the host's ability to eliminate M.tb. Therefore, a small-size granuloma may favor host defense whereas a relatively large-size counterpart may favor the persistence of mycobacterial bacilli, regardless of the magnitude of $\mathrm{T}$ cells generated. The current limitation to diagnostic imaging makes studying the evolution of the granuloma in latently infected humans difficult and as a consequence, little is known about how its structure changes over the course of infection in otherwise healthy individuals.

\section{THE "IMMUNE GRANULOMA": A NICHE FOR BACTERIAL PERSISTENCE}

Regardless of whether the granuloma functions to limit bacterial dissemination, much evidence suggests that $M$.tb is especially adept at altering the immune response within the granuloma, creating a uniquely suppressed environment largely through the induction of IL-10 (de Waal Malefyt et al., 1993; Chiu et al., 2007, 2008; Higgins et al., 2009; Marino et al., 2010; Redford et al., 2010;
O'leary et al., 2011; Shaler et al., 2011). Functionally, the infected macrophages within the granuloma are altered, showing a reduced capacity to produce bactericidal products such as nitric oxide, while showing enhanced IL-10 production (de Waal Malefyt et al., 1993; Chiu et al., 2007, 2008; Higgins et al., 2009; Marino et al., 2010; Redford et al., 2010; O’leary et al., 2011; Shaler et al., 2011). Interestingly, while the macrophage populations of the granuloma have reduced bactericidal function, they continue to produce large amounts of chemokines facilitating the continuous recruitment APC populations into the granuloma (Schreiber et al., 2010; Shaler et al., 2011). Recent studies utilizing intravital microscopy have revealed significant movement of inflammatory APCs both into and out of the granuloma (Schreiber etal., 2011). It is this movement of infected APCs that has been speculated to facilitate the early dissemination of M.tb. Likewise, human granuloma contains a high frequency of foxp3 $+\mathrm{T}$ regulatory cells (Rahman, 2009). In addition, murine studies have confirmed that $T$ cells residing within the granuloma display a highly altered, and functionally suppressed phenotype. Despite the central role of IL-10 in suppressing $\mathrm{T}$ cell and macrophage activation within the granuloma, IL-10 neutralization or infection of IL-10 knockout (KO) mice results in only marginally reduced bacterial loads (de Waal Malefyt et al., 1993; Jacobs et al., 2002; Chiu et al., 2007; Higgins et al., 2009). Given M.tb's long evolution with humans, it is not surprising that M.tb targets multiple pathways to interrupt the host immune response. Moreover, while conventionally immune suppression would appear to benefit only the pathogen, the induction of IL-10 may actually be a host-mediated event required to limit unwanted immunopathology.

\section{THE "CHRONIC GRANULOMA": A DYNAMIC INTERPLAY BETWEEN PERSISTING M.tb AND THE HOST IMMUNE RESPONSE}

Following the establishment of the "immune granuloma," a period of immune quiescence is established. It is during this stage that chronic immune activation leads to significant alterations in the morphology and functionality of the granuloma, many of which are not typically seen in mice. Post-mortem studies of latently infected humans and non-human primates have revealed that the evolution of the granuloma structure is highly divergent, not only between individuals, but even within a single individual (Capuano et al., 2003; Flynn et al., 2003; Barry et al., 2009). Typically, within an infected individual, a spectrum of granuloma structures are seen. For instance, both the fully calcified lesions containing no bacilli and the fibrotically encapsulated necrotic granulomas containing large numbers of live bacteria can be seen in the lung of the same individual, indicating that the evolution of the granuloma is a highly dynamic process (Capuano et al., 2003; Flynn et al., 2003; Barry et al., 2009).

While the granuloma has long been believed to be a protective host's response, it is now acknowledged to result from a dynamic and continuous interplay between the host's immune response and persisting M.tb. The continuous "battle" between chronic immune activation and bacterial persistence causes infected macrophages to adopt an irregular epithelial-like or "epithelioid" appearance, and to fuse together forming multinucleated giant cells in the core of granuloma (Hunter et al., 2007; Hunter, 2011). Indeed, the virulent Mycobacterium has been shown to induce macrophage cell 
death frequently throughout the course of infection, which several studies have shown to be a potential mechanism of gaining access to new host macrophages (Davis and Ramakrishnan, 2009). Newly recruited macrophages quickly phagocytose the dead bodies and become saturated with mycobacteria and lipids (Peyron et al., 2008). These lipid-rich macrophages that accumulate within the granuloma are known as foamy macrophages due to their distinct appearance, and are now recognized as a major contributing factor in the persistence of M.tb (Peyron et al., 2008). Recent studies have shown that foamy macrophages isolated from humans have lost key functions including their ability to phagocytose and to produce essential bactericidal agents such as nitric oxide (Peyron et al., 2008). Moreover, the polarization of macrophage populations within the granuloma are thought to shift from being a classically activated (M1) population toward that of an alternatively activated (M2), with reduced bactericidal capacity (Redente et al., 2010). Thus, such foamy macrophages have been proposed to function as the reservoirs of $M$.t $b$ whereby the bacterium is able to successfully manipulate the infected macrophage into not only a safe haven but also a source of nutrients required for the synthesis of its cell wall and replication.An infected host typically houses a highly heterogeneous mixture of granuloma types ranging from large necrotic granulomas containing large amounts of bacilli, to completely calcified structures devoid of any detectible bacteria (Turner et al., 2003; Hunter et al., 2007; Hunter, 2011). In the later stages of granuloma evolution, fibrotic encapsulation can be seen in cases of both active and latent infection. Currently, it is unclear whether encapsulation functions to prevent bacterial escape, or to limit immune infiltration into the granuloma. Although complete mycobacterial clearance is rarely seen, latently infected humans display the evidence of healed granulomas, marked by central calcification in conjunction with fibrotic encapsulation containing no detectible bacilli (Opie, 1917). Despite the observed absence of bacteria within highly calcified granulomas, it is currently unclear whether this represents immune-mediated clearance, or simply a structural artifact left behind following M.tb escape.

\section{Mycobacterium tuberculosis FACILITATES PERSON-TO-PERSON TRANSMISSION THROUGH ALTERATIONS TO THE GRANULOMA}

Despite the attempts of the host to contain M.tb within the granuloma, as the infection progresses, the majority of individuals will develop granulomas with a necrotic focus formed due to the caesation of the macrophage infused center (Kim et al., 2010; Figure 2). This eventual necrosis of the granuloma is now accepted as a necessary event in facilitating the transmission of M.tb by disrupting the lung structure and allowing $M$.tb to gain access to the major airways. It should be noted that, in addition to person-toperson transmission, the active granuloma leaking bacteria into the airways may also allow for intrapulmonary dissemination (Cardona, 2009, 2010; Cardona and Ivanyi, 2011). It is therefore likely that the heterogeneity in granuloma structure seen in different lung regions of the same host represents different evolutionary timelines. Although much remains to be understood, it is clear that the evolution of granuloma is the result of the dynamic interplay between persisting mycobacteria and the host immune response, continuously evolving throughout the course of M.tb infection. Interestingly, M.tb may actually utilize the host

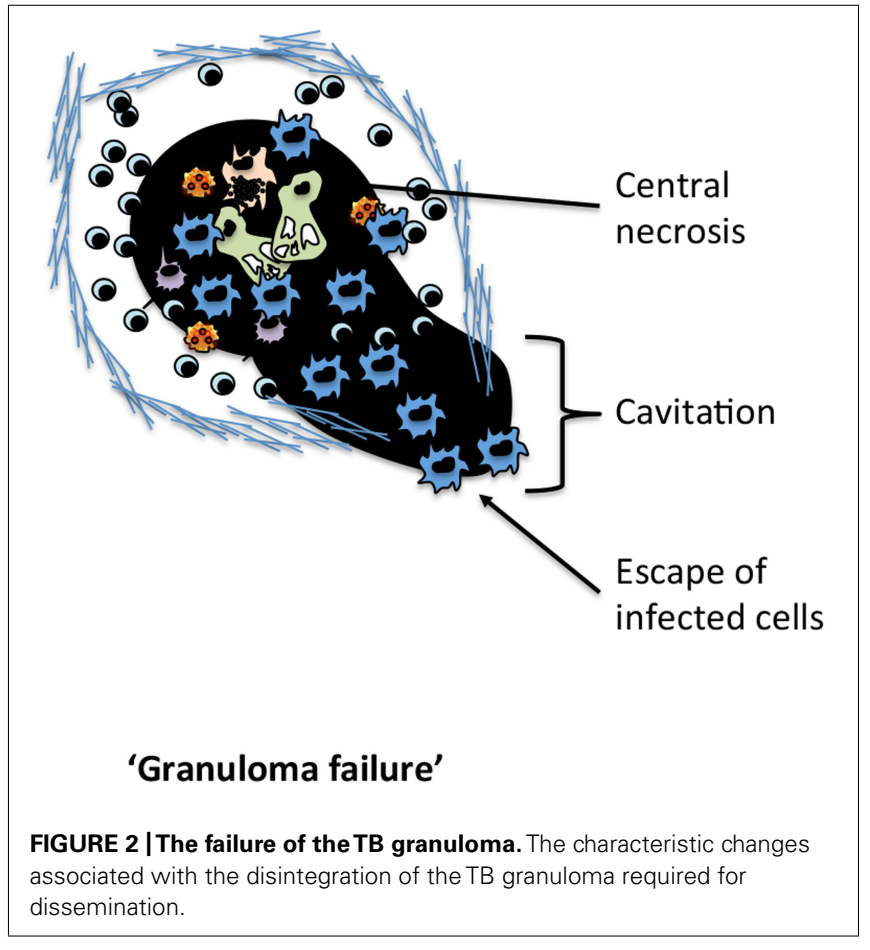

immune response to facilitate the structural changes required to facilitate person-to-person transmission. In line with this, while essential to preventing bacterial dissemination, paradoxically IFN$\gamma$-producing Th1 cells may also play an integrate role in facilitating bacterial transmission (Ehlers et al., 2001). The ability of M.tb to manipulate the host immune response as means to facilitate central granuloma necrosis and facilitate its transmission while deterring immune-mediated bacterial clearance is a remarkable but poorly understood feature of M.tb. It is important to note that the processes of bacterial dissemination within a host, and transmission between hosts may be independently regulated. For instance, clinically it has been observed that despite exaggerated bacterial burdens and extensive dissemination, HIV-AIDS individuals co-infected with $M . t b$ transmit M.tb person-to-person far less efficiently (Doenhoff, 1997; Ledru et al., 1999; Corbett et al., 2003; Glynn et al., 2008). The inability of the HIV-infected host to spread M.tb has been attributed to a failure of M.tb to drive central granuloma necrosis and cavitation, and the transport of bacilli to the airway. These observations argue that M.tb utilizes the necrotic granuloma as a portal for person-to-person transmission.

While it is well-known that changes to the granuloma structure are required for bacterial transmission, it is currently unknown whether the host or bacteria are responsible for these changes. Recently, studies have examined the granuloma at early and chronic stages of disease revealing a dramatic shift in the genes expressed by both $M . t b$ and the host immune response. Notably, within the granuloma, the host immune response shifts from predominately pro-inflammatory during the early phases of infection, to immunosuppressive during the chronic stages (Karakousis et al., 2004; Ly et al., 2007; Mehra et al., 2010). Coincidentally, M.tb expresses a defined set of genes that function to facilitate immune 
activation, while simultaneously expressing enzymes to combat immune-mediated clearance (Rohde et al., 2012). This is later followed by a shift in gene expression thought to facilitate immune senescence within the granuloma, allowing for M.tb's persistence (Rohde etal., 2012). While traditionally M.tb is thought to lie dominant, it has recently been demonstrated that throughout the course infection M.tb will periodically “awaken," up-regulating a number genes and sample the immune environment (Karakousis et al., 2004). This sampling allows M.tb to identify the optimal conditions for facilitating person-to-person transmission. Moreover, during the caseous stage of granuloma formation there is a further shift in the genes expressed by M.tb with a significant up-regulation of genes associated with lipid metabolism (Kim et al., 2010). Notably, most mycobacterial species are rich in immunomodulatory lipids, which play a central role in immune evasion. Intriguingly, the distribution and release of certain lipids by $M . t b$ varies significantly over the course of infection, providing a means by which M.tb directs the host immune response. To this end, M.tb can release toxic lipids and generate targeted tissue damage. The generation of central necrosis is essential to facilitating cavitation and promoting $M$.t $b$ transmission. Trehalose 6,6 $6^{\prime}$-dimycolate (cord factor) has potent cytotoxic effects and has been implicated in the generation of central necrosis of the granuloma and the transmission of M.tb (Hunter et al., 2006; Kim et al., 2010). Recently, it has been shown that neutrophils and AMs recognize mycobacterial cord factor through their surface ctype lectin receptor, mincle (Behler et al., 2012; Lee et al., 2012). The engagement of mincle leads to a pro-inflammatory cytokine pathway that aids in the early cellular recruitment and control of mycobacteria (Behler et al., 2012; Lee et al., 2012). Interestingly, however, the proportion of cord factor varies greatly throughout

\section{REFERENCES}

Algood, H. M., Lin, P. L., and Flynn, J. L. (2005). Tumor necrosis factor and chemokine interactions in the formation and maintenance of granulomas in tuberculosis. Clin. Infect. Dis. 41, S189-S193.

Anis, M. M., Fulton, S. A., Reba, S. M., Liu, Y., Harding, C. V., and Boom, W. H. (2008). Modulation of pulmonary dendritic cell function during mycobacterial infection. Infect. Immun. 76, 671-677.

Aston, C., Rom, W. N., Talbot, A. T., and Reibman, J. (1998). Early inhibition of mycobacterial growth by human alveolar macrophages is not due to nitric oxide. Am. J. Respir. Crit. Care Med. 157, 1943-1950.

Barry, C. E. III, Boshoff, H. I., Dartois, V., Dick, T., Ehrt, S., Flynn, J., et al. (2009). The spectrum of latent tuberculosis: rethinking the biology and intervention strategies. Nat. Rev. Microbiol. 7, 845-855.

Beham, A. W., Puellmann, K., Laird, R., Fuchs, T., Streich, R., Breysach, C., et al. (2011). A TNF-regulated recombinatorial macrophage immune receptor implicated in granuloma formation in tuberculosis. PLoS Pathog. 7:e1002375. doi: 10.1371/journal.ppat.1002375

Behler, F., Steinwede, K., Balboa, L., Ueberberg, B., Maus, R., Kirchhof, G., etal. (2012). Role of Mincle in alveolar macrophage-dependent innate immunity against mycobacterial infections in mice. J. Immunol. 189, 3121-3129.

Birkness, K. A., Guarner, J., Sable, S. B., Tripp, R. A., Kellar, K. L., Bartlett, J., et al. (2007). An in vitro model of the leukocyte interactions associated with granuloma formation in Mycobacterium tuberculosis infection. Immunol. Cell Biol. 85, 160-168.

Capuano, S. V. III, Croix, D. A., Pawar, S., Zinovik, A., Myers, A., Lin, P. L., et al. (2003). Experimental Mycobacterium tuberculosis infection of cynomolgus macaques closely resembles the various manifestations of human $M$. tuberculosis infection. Infect. Immun. 71, 5831-5844.

Cardona, P. J. (2009). A dynamic reinfection hypothesis of latent tuberculosis infection. Infection 37, 80-86.

the course of infection with its synthesis heavily up-regulated by $M . t b$ during the development of central necrosis and cavitation (Hunter et al., 2006; Kim et al., 2010). Indeed, studies have linked the amount of cord factor released by M.tb to the extent of necrosis and cavity formation (Hunter et al., 2006; Kim et al., 2010). Moreover, previous studies have documented mincle as a key receptor in the detection of necrosis and the development of an inflammatory response upon tissue damage (Yamasaki et al., 2008). Given that cord factor is known for its cytotoxic effects, one may speculated that engaging mincle may be central to the development of a pro-inflammatory response capable of aiding the formation of cavitation within the granuloma. Utilizing the host immune machinery M.tb facilitates the necessary structural changes to ensure its own transmission, which occurs at a time when the immune system is most vulnerable.

\section{CONCLUDING REMARKS}

While the true nature of the granuloma still remains to be defined, it is now clearly evident that the granuloma is not just a hostmediated entity of segregation and rather, it is a dynamic battlefield bearing the scars left both by the pathogen and the host immune response. While it may have been originally destined to restrain bacterial dissemination, M.tb efficiently hijacks the granuloma to provoke the generation of an immunologically sheltered niche to reside within and persist until the situation is favorable to bacterial transmission.

\section{ACKNOWLEDGMENTS}

The work cited from the authors' laboratory was supported by funds from the Canadian Institutes of Health Research and the Government of Ontario.

Cardona, P. J. (2010). Revisiting the natural history of tuberculosis. The inclusion of constant reinfection, host tolerance, and damage-response frameworks leads to a better understanding of latent infection and its evolution towards active disease. Arch. Immunol. Ther. Exp. 58, 7-14.

Cardona, P. J., and Ivanyi, J. (2011). The secret trumps, impelling the pathogenicity of tubercle bacilli. Enferm. Infecc. Microbiol. Clin. 29(Suppl. 1), 14-19.

Castano, D., Barrera, L. F., and Rojas, M. (2011). Mycobacterium tuberculosis alters the differentiation of monocytes into macrophages in vitro. Cell. Immunol. 268, 60-67.

Chiu, B. C., Freeman, C. M., Stolberg, V. R., Hu, J. S., Komuniecki, E., and Chensue, S. W. (2004). The innate pulmonary granuloma: characterization and demonstration of dendritic cell recruitment and function. Am. J. Pathol. 164, 1021-1030.

Chiu, B. C., Stolberg, V. R., and Chensue, S. W. (2008). Mononuclear phagocyte-derived IL-10 suppresses the innate IL-12/
IFN-gamma axis in lung-challenged aged mice. J. Immunol. 181, 31563166 .

Chiu, B. C., Stolberg, V. R., Freeman, C. M., and Chensue, S. W. (2007). Mononuclear phagocytederived interleukin-10 suppresses the innate pulmonary granuloma cytokine response in aged mice. Am. J. Pathol. 171, 829-837.

Corbett, E. L., Watt, C. J., Walker, N., Maher, D., Williams, B. G., Raviglione, M. C., etal. (2003). The growing burden of tuberculosis: global trends and interactions with the HIV epidemic. Arch. Intern. Med. 163, 1009-1021.

Davis, J. M., and Ramakrishnan, L. (2009). The role of the granuloma in expansion and dissemination of early tuberculous infection. Cell 136, 37-49.

Dejerine, J. (1884). Recherche du bacilli de koch dans la tuberculose calcifee. Compt. Rend. Soc. Biol. 36, 500-505.

de le Boe, F. (1679). Opera medica, Vol. 934. Amstelodami: Apud Danielem Elsevirium, et Abrahamum Wolfgang, 150-200. 
de Waal Malefyt, R., Yssel, H., and De Vries, J. E. (1993). Direct effects of IL-10 on subsets of human CD4+ T cell clones and resting $\mathrm{T}$ cells. Specific inhibition of IL-2 production and proliferation. J. Immunol. 150, 4754-4765.

Dlugovitzky, D., Bay, M. L., Rateni, L., Fiorenza, G., Vietti, L., Farroni, M. A., et al. (2000). Influence of disease severity on nitrite and cytokine production by peripheral blood mononuclear cells (PBMC) from patients with pulmonary tuberculosis (TB). Clin. Exp. Immunol. 122, 343-349.

Doenhoff, M. J. (1997). A role for granulomatous inflammation in the transmission of infectious disease: schistosomiasis and tuberculosis. Parasitology 115(Suppl.), S113S125.

Egen, J. G., Rothfuchs, A. G., Feng, C. G., Horwitz, M. A., Sher, A., and Germain, R. N. (2011). Intravital imaging reveals limited antigen presentation and $\mathrm{T}$ cell effector function in mycobacterial granulomas. Immunity 34, 807-819.

Egen, J. G., Rothfuchs, A. G., Feng, C. G., Winter, N., Sher, A., and Germain, R. N. (2008). Macrophage and T cell dynamics during the development and disintegration of mycobacterial granulomas. Immunity 28, 271-284.

Ehlers, S., Benini, J., Held, H. D., Roeck, C., Alber, G., and Uhlig, S. (2001). Alphabeta $\mathrm{T}$ cell receptor-positive cells and interferon-gamma, but not inducible nitric oxide synthase, are critical for granuloma necrosis in a mouse model of mycobacteriainduced pulmonary immunopathology. J. Exp. Med. 194, 1847-1859.

Feldman, W., and Baggenstoss, A. (1938). The residual infectivity of the primary complex of tuberculosis. Am. J. Pathol. 14, 473-490.

Feldman, W., and Baggenstoss, A. (1939). The occurence of virulent tubercle bacilli in presumably nontuberculous lung tissue. Am. J. Pathol. 15, 501-515.

Ferrer, N. L., Gomez, A. B., Neyrolles, O., Gicquel, B., and Martin, C. (2010). Interactions of attenuated Mycobacterium tuberculosis phoP mutant with human macrophages. PLoS ONE 5:e12978. doi: 10.1371/journal.pone.0012978

Flynn, J. L., Capuano, S. V., Croix, D., Pawar, S., Myers, A., Zinovik, A., et al. (2003). Non-human primates: a model for tuberculosis research. Tuberculosis 83, 116-118.

Flynn, J. L., Scanga, C. A., Tanaka, K. E., and Chan, J. (1998). Effects of aminoguanidine on latent murine tuberculosis. J. Immunol. 160, 17961803.

Gallegos, A. M., Van Heijst, J. W., Samstein, M., Su, X., Pamer, E. G., and Glickman, M. S. (2011). A gamma interferon independent mechanism of CD4 T cell mediated control of $M$. tuberculosis infection in vivo. PLoS Pathog. 7:e1002052. doi: 10.1371/journal.ppat.1002052

Geldmacher, C., Zumla, A., and Hoelscher, M. (2012). Interaction between HIV and Mycobacterium tuberculosis: HIV-1-induced CD4 Tcell depletion and the development of active tuberculosis. Curr. Opin. HIV AIDS 7, 268-275.

Glynn, J. R., Murray, J., Bester, A. Nelson, G., Shearer, S., and Sonnenberg, P. (2008). Effects of duration of HIV infection and secondary tuberculosis transmission on tuberculosis incidence in the South African gold mines. AIDS 22, 1859-1867.

Higgins, D. M., Sanchez-Campillo, J., Rosas-Taraco, A. G., Lee, E. J., Orme, I. M., and Gonzalez-Juarrero, M. (2009). Lack of IL-10 alters inflammatory and immune responses during pulmonary Mycobacterium tuberculosis infection. Tuberculosis (Edinb) 89, 149-157.

Hunter, R. L. (2011). Pathology of post primary tuberculosis of the lung: an illustrated critical review. Tuberculosis 91, 497-509.

Hunter, R. L., Jagannath, C., and Actor, J. K. (2007). Pathology of postprimary tuberculosis in humans and mice: contradiction of long-held beliefs. Tuberculosis 87 , 267-278.

Hunter, R. L., Olsen, M., Jagannath, C., and Actor, J. K. (2006). Trehalose 6,6'-dimycolate and lipid in the pathogenesis of caseating granulomas of tuberculosis in mice. Am. J.Pathol. 168, 1249-1261.

Jacobs, M., Fick, L., Allie, N., Brown, N., and Ryffel, B. (2002). Enhanced immune response in Mycobacterium bovis bacille calmette guerin (BCG)-infected IL-10-deficient mice. Clin. Chem. Lab. Med. 40, 893-902.

Jagannath, C., Actor, J. K., and Hunter, R. L. Jr. (1998). Induction of nitric oxide in human monocytes and monocyte cell lines by Mycobacterium tuberculosis. Nitric Oxide 2, 174-186.

Johnson, C. M., Cooper, A. M., Frank, A. A., and Orme, I. M. (1998). Adequate expression of protective immunity in the absence of granuloma formation in Mycobacterium tuberculosis-infected mice with a disruption in the intracellular adhesion molecule 1 gene. Infect. Immun. 66, 1666-1670.

Kaneko, H., Yamada, H., Mizuno, S., Udagawa, T., Kazumi, Y., Sekikawa, K., etal. (1999). Role of tumor necrosis factor-alpha in Mycobacterium-induced granuloma formation in tumor necrosis factoralpha-deficient mice. Lab. Invest. 79, 379-386.

Karakousis, P. C., Yoshimatsu, T., Lamichhane, G., Woolwine, S. C., Nuermberger, E. L., Grosset, J., et al. (2004). Dormancy phenotype displayed by extracellular Mycobacterium tuberculosis within artificial granulomas in mice. J. Exp. Med. 200, 647-657.

Kim, M. J., Wainwright, H. C., Locketz, M., Bekker, L. G., Walther, G. B. Dittrich, C., et al. (2010). Caseation of human tuberculosis granulomas correlates with elevated host lipid metabolism. EMBO Mol. Med. 2, 258-274.

Ledru, E., Ledru, S., and Zoubga, A. (1999). Granuloma formation and tuberculosis transmission in HIVinfected patients. Immunol. Today 20 336-337.

Lee, W. B., Kang, J. S., Yan, J. J., Lee, M. S., Jeon, B. Y., Cho, S. N., et al. (2012). Neutrophils promote Mycobacterial trehalose dimycolate-induced lung inflammation via the mincle pathway. PLoS Pathog. 8:e1002614. doi: 10.1371/journal.ppat.1002614

Ly, L. H., Russell, M. I., and Mcmurray, D. N. (2007). Microdissection of the cytokine milieu of pulmonary granulomas from tuberculous guinea pigs. Cell. Microbiol. 9, 1127-1136.

Marino, S., Myers, A., Flynn, J. L., and Kirschner, D. E. (2010). TNF and IL-10 are major factors in modulation of the phagocytic cell environment in lung and lymph node in tuberculosis: a next-generation twocompartmental model. J. Theor. Biol. 265, 586-598.

Mehra, S., Pahar, B., Dutta, N. K. Conerly, C. N., Philippi-Falkenstein, K., Alvarez, X., et al. (2010). Transcriptional reprogramming in nonhuman primate (rhesus macaque) tuberculosis granulomas. PLOS ONE 5:e12266. doi: 10.1371/journal.pone. 0012266

Mogues, T., Goodrich, M. E., Ryan, L., Lacourse, R., and North, R. J. (2001). The relative importance of $\mathrm{T}$ cell subsets in immunity and immunopathology of airborne Mycobacterium tuberculosis infection in mice. J. Exp. Med. 193, 271-280.

O’leary, S., O’Sullivan, M. P., and Keane, J. (2011). IL-10 blocks phagosome maturation in mycobacterium tuberculosis-infected human macrophages. Am. J. Respir. Cell Mol. Biol. 45, 172-180.

Opie, E., and Aronson, J. (1927). Tubercle bacilli in latent tuberculosis lesions and in lung tissue without tuberculous lesions. Arch. Pathol. Lab. Med. 4, 1-21.

Opie, E. L. (1917). The relation of apical tuberculosis of adults to the focal tuberculosis of children. J. Exp. Med. 26, 263-277.

Pearl, J. E., Khader, S. A., Solache, A., Gilmartin, L., Ghilardi, N., Desauvage, F., et al. (2004). IL27 signaling compromises control of bacterial growth in mycobacteriainfected mice. J. Immunol. 173, 74907496.

Peyron, P., Vaubourgeix, J., Poquet, Y., Levillain, F., Botanch, C., Bardou, F., et al. (2008). Foamy macrophages from tuberculous patients' granulomas constitute a nutrient-rich reservoir for $M$. tuberculosis persistence. PLoS Pathog. 4:e1000204. doi: 10.1371/journal.ppat.1000204

Rahman, S. (2009). Compartmentalization of immune responses in human tuberculosis: few CD8+ effector T cells but elevated levels of FoxP3+ regulatory $\mathrm{t}$ cells in the granulomatous lesions. Am. J. Pathol. 174, 2211-2224.

Ramakrishnan, L. (2012). Revisiting the role of the granuloma in tuberculosis. Nat. Rev. Immunol. 12, 352-366.

Redente, E. F., Higgins, D. M., DwyerNield, L. D., Orme, I. M., GonzalezJuarrero, M., and Malkinson, A. M. (2010). Differential polarization of alveolar macrophages and bone marrow-derived monocytes following chemically and pathogeninduced chronic lung inflammation. J. Leukoc. Biol. 88, 159-168.

Redford, P. S., Boonstra, A., Read, S., Pitt, J., Graham, C., Stavropoulos, E., et al. (2010). Enhanced protection to Mycobacterium tuberculosis infection in IL-10-deficient mice is accompanied by early and enhanced Th1 responses in the lung. Eur. J. Immunol. 40, 2200-2210.

Rhoades, E. R., Frank, A. A., and Orme, I. M. (1997). Progression of chronic pulmonary tuberculosis in mice aerogenically infected with virulent Mycobacterium tuberculosis. Tuber. Lung Dis. 78, 57-66.

Rich, E. A., Torres, M., Sada, E., Finegan, C. K., Hamilton, B. D., and Toossi, Z. (1997). Mycobacterium tuberculosis (MTB)-stimulated production of nitric oxide by human alveolar macrophages and relationship of nitric oxide production to 
growth inhibition of MTB. Tuber. Lung Dis. 78, 247-255.

Robertson, H. (1933). The persistence of tuberculous infections. Am. J. Pathol. 9, 711-718.

Rohde, K. H., Veiga, D. F., Caldwell, S., Balazsi, G., and Russell, D. G. (2012). Linking the transcriptional profiles and the physiological states of Mycobacterium tuberculosis during an extended intracellular infection. PLoS Pathog. 8:e1002769. doi: 10.1371/journal.ppat.1002769

Saunders, B. M., Frank, A. A., Orme, I. M., and Cooper, A. M. (2002). CD4 is required for the development of a protective granulomatous response to pulmonary tuberculosis. Cell. Immunol. 216, 65-72.

Schreiber, H. A., Harding, J. S., Hunt, O., Altamirano, C. J., Hulseberg, P. D., Stewart, D., et al. (2011). Inflammatory dendritic cells migrate in and out of transplanted chronic mycobacterial granulomas in mice. J. Clin. Invest. 121, 3902-3913.

Schreiber, H. A., Hulseberg, P. D. Lee, J., Prechl, J., Barta, P., Szlavik, N., et al. (2010). Dendritic cells in chronic mycobacterial granulomas restrict local anti-bacterial $\mathrm{T}$ cell response in a murine model. PLoS ONE 5:e11453. doi: 10.1371/journal.pone. 0011453

Scott, H. M., and Flynn, J. L. (2002). Mycobacterium tuberculosis in chemokine receptor 2-deficient mice: influence of dose on disease progression. Infect. Immun. 70, 59465954.

Scott-Browne, J. P., Shafiani, S., Tucker-Heard, G., Ishida-Tsubota, K., Fontenot, J. D., Rudensky, A. Y., et al.
(2007). Expansion and function of Foxp3-expressing $\mathrm{T}$ regulatory cells during tuberculosis. J. Exp. Med. 204, 2159-2169.

Segovia-Juarez, J. L., Ganguli, S., and Kirschner, D. (2004). Identifying control mechanisms of granuloma formation during $M$. tuberculosis infection using an agent-based model. J. Theor. Biol. 231, 357-376.

Shaler, C. R., Horvath, C., Lai, R. and Xing, Z. (2012). Understanding delayed $\mathrm{T}$-cell priming, lung recruitment, and airway luminal $\mathrm{T}$ cell responses in host defense against pulmonary tuberculosis. Clin. Dev. Immunol. 2012, 1-13.

Shaler, C. R., Kugathasan, K., Mccormick, S., Damjanovic, D., Horvath, C., Small, C. L., et al. (2011). Pulmonary mycobacterial granuloma increased IL-10 production contributes to establishing a symbiotic host-microbe microenvironment. Am. J. Pathol. 178, 1622 1634.

Taylor, J. L., Hattle, J. M., Dreitz, S. A., Troudt, J. M., Izzo, L. S., Basaraba, R. J., et al. (2006). Role for matrix metalloproteinase 9 in granuloma formation during pulmonary Mycobacterium tuberculosis infection. Infect. Immun. 74, 61356144.

Tsai, M. C., Chakravarty, S., Zhu, G., Xu, J., Tanaka, K., Koch, C., et al. (2006). Characterization of the tuberculous granuloma in murine and human lungs: cellular composition and relative tissue oxygen tension. Cell. Microbiol. 8, 218-232.

Turner, O. C., Basaraba, R. J., and Orme, I. M. (2003). Immunopathogenesis of pulmonary granulomas in the guinea pig after infection with Mycobacterium tuberculosis. Infect. Immun. 71, 864-871.

Via, L. E., Lin, P. L., Ray, S. M., Carrillo, J., Allen, S. S., Eum, S. Y., et al. (2008). Tuberculous granulomas are hypoxic in guinea pigs, rabbits, and nonhuman primates. Infect. Immun 76, 2333-2340.

Volkman, H. E., Clay, H., Beery, D., Chang, J. C. W., Sherman, D. R., and Ramakrishnan, L. (2004) Tuberculous granuloma formation is enhanced by a Mycobacterium virulence determinant. PLoS Biol. 2:e367. doi: 10.1371/journal.pbio. 0020367

Volkman, H. E., Pozos, T. C., Zheng, J., Davis, J. M., Rawls, J. F., and Ramakrishnan, L. (2010). Tuberculous granuloma induction via interaction of a bacterial secreted protein with host epithelium. Science 327 466-469.

Wang, C. (1916). An experimentalstudy of latent tuberculosis. Lancet 2 , 417-419.

Welin, A., Raffetseder, J., Eklund, D., Stendahl, O., and Lerm, M. (2011). Importance of phagosomal functionality for growth restriction of Mycobacterium tuberculosis in primary human macrophages. J. Innate Immun. 3, 508-518.

WHO. (2012). Global Tuberculosis Report 2012. Geneva: WHO.

Winau, F., Weber, S., Sad, S., De Diego, J., Hoops, S. L., Breiden, B., et al. (2006). Apoptotic vesicles crossprime CD8 $\mathrm{T}$ cells and protect against tuberculosis. Immunity 24 105-117.

Yahagi, A., Umemura, M., Tamura, T., Kariyone, A., Begum, M. D.,
Kawakami, K., et al. (2010). Suppressed induction of mycobacterial antigen-specific Th1-type CD4+ $\mathrm{T}$ cells in the lung after pulmonary mycobacterial infection. Int. Immunol. 22, 307-318.

Yamasaki, S., Ishikawa, E., Sakuma, M., Hara, H., Ogata, K., and Saito, T. (2008). Mincle is an ITAM-coupled activating receptor that senses damaged cells. Nat. Immunol. 9, 11791188 .

Conflict of Interest Statement: The authors declare that the research was conducted in the absence of any commercial or financial relationships that could be construed as a potential conflict of interest.

Received: 03 November 2012; paper pending published: 13 December 2012; accepted: 25 January 2013; published online: 14 February 2013.

Citation: Shaler CR, Horvath CN, Jeyanathan M and Xing Z (2013) Within the Enemy's Camp: contribution of the granuloma to the dissemination, persistence and transmission of Mycobacterium tuberculosis. Front. Immun. 4:30. doi: 10.3389/fimmu.2013.00030

This article was submitted to Frontiers in Inflammation, a specialty of Frontiers in Immunology.

Copyright (c) 2013 Shaler, Horvath, Jeyanathan and Xing. This is an openaccess article distributed under the terms of the Creative Commons Attribution License, which permits use, distribution and reproduction in other forums, provided the original authors and source are credited and subject to any copyright notices concerning any third-party graphics etc. 